\title{
Pion vector and scalar form factors with dynamical overlap quarks
}

\section{JLQCD and TWQCD collaborations: T. Kaneko ${ }^{\star a, b} \dagger$ S. Aoki ${ }^{c, d}$, T. W. Chiu ${ }^{e}$, H. Fukaya ${ }^{a, f}$, S. Hashimoto ${ }^{a, b}$, T. H. Hsieh ${ }^{g}$, H. Matsufuru ${ }^{a}$, J. Noaki ${ }^{a}$, T. Onogi ${ }^{h}$, E. Shintani ${ }^{a}$ and N. Yamada ${ }^{a, b}$}

${ }^{a}$ High Energy Accelerator Research Organization (KEK), Ibaraki 305-0801, Japan

${ }^{b}$ School of High Energy Accelerator Science, The Graduate University for Advanced Studies (Sokendai), Ibaraki 305-0801, Japan

${ }^{c}$ Graduate School of Pure and Applied Sciences, University of Tsukuba, Ibaraki 305-8571, Japan

${ }^{d}$ Riken BNL Research Center, Brookhaven National Laboratory, Upton, New York 11973, USA

e Physics Department, Center for Theoretical Sciences, and Center for Quantum Science and Engineering, National Taiwan University, Taipei, 10617, Taiwan

${ }^{f}$ The Niels Bohr Institute, The Niels Bohr International Academy, Blegdamsvej 17 DK-2100 Copenhagen $\emptyset$, Denmark

${ }^{g}$ Research Center for Applied Sciences, Academia Sinica, Taipei 115, Taiwan

${ }^{h}$ Yukawa Institute for Theoretical Physics, Kyoto University, Kyoto 606-8502, Japan

We calculate the pion vector and scalar form factors in two-flavor QCD. Gauge configurations are generated with dynamical overlap quarks on a $16^{3} \times 32$ lattice at a lattice spacing of $0.12 \mathrm{fm}$ with sea quark masses down to a sixth of the physical strange quark mass. Contributions of disconnected diagrams to the scalar form factor is calculated employing the all-to-all quark propagators. We present a detailed comparison of the vector and scalar radii with chiral perturbation theory to two loops.

The XXVI International Symposium on Lattice Field Theory

July 14-192008

Williamsburg, Virginia, USA

\footnotetext{
*Speaker.

${ }^{\dagger}$ E-mail: takashi.kaneko@kek.jp
} 


\section{Introduction}

Pion electromagnetic form factor $F_{V}\left(q^{2}\right)$ is one of the fundamental observables in hadron physics. An analysis of experimental data based on chiral perturbation theory (ChPT) at two loops leads to a precise estimate of the charge radius $\left\langle r^{2}\right\rangle_{V}$ [1]. A detailed comparison of $\left\langle r^{2}\right\rangle_{V}$ between $\mathrm{ChPT}$ and non-perturbative calculations on the lattice may provide a good testing ground for recent lattice simulations in the chiral regime as well as a better understanding of the chiral behavior of $F_{V}\left(q^{2}\right)$.

While there is no experimental processes directly related to the scalar form factor $F_{S}\left(q^{2}\right)$, the chiral behavior of the scalar radius $\left\langle r^{2}\right\rangle_{S}$ is interesting, as it provides a determination of the LEC $l_{4}$ and has a 6 times enhanced chiral logarithm compared to $\left\langle r^{2}\right\rangle_{V}$. A non-perturbative determination on the lattice is challenging, because we need to evaluate disconnected three-point functions.

In this article, we update our analysis of $F_{V}\left(q^{2}\right)$ reported at the last conference [2] with doubled statistics, and present newly obtained results for $F_{S}\left(q^{2}\right)$. These quantities are measured on gauge configurations of two-flavor QCD on a $16^{3} \times 32$ lattice generated with the overlap quark action along the fixed topology strategy [3]. The lattice spacing determined from the Sommer scale $r_{0}=0.49 \mathrm{fm}$ is $a=0.1184(21) \mathrm{fm}$. We refer the reader to Refs. $[4,5]$ for detailed setup and overviews of our production simulations.

\section{Measurement of pion correlation functions}

We measure pion correlators through all-to-all quark propagators [6]. Contributions of 100 low-lying modes $\left(\lambda^{(k)}, u^{(k)}\right)\left(k=1, \ldots, N_{\mathrm{ep}} ; N_{\mathrm{ep}}=100\right)$ of the overlap operator $D$ are evaluated exactly, whereas the remaining high modes are taken into account stochastically by the $Z_{2}$ noise method. We prepare a single noise vector for each configuration, and dilute [6] it into $N_{d}=$ $3 \times 4 \times N_{t} / 2$ vectors $\eta^{(k)}\left(k=1, \ldots, N_{d}\right)$ with support on a single value for color and spinor indices and at two time-slices. The all-to-all propagator can then be expressed in a simple form $D^{-1}=\sum_{k=1}^{N_{\text {vec }}} v^{(k)} w^{(k) \dagger}\left(N_{\mathrm{vec}}=N_{\mathrm{ep}}+N_{d}\right)$ with two set of vectors

$$
v^{(k)}=\left\{\frac{u^{(1)}}{\lambda^{(1)}}, \ldots, \frac{u^{\left(N_{\mathrm{ep}}\right)}}{\lambda^{\left(N_{\mathrm{ep}}\right)}}, x^{(1)}, \ldots, x^{\left(N_{d}\right)}\right\}, \quad w^{(k)}=\left\{u^{(1)}, \ldots, u^{\left(N_{\mathrm{ep}}\right)}, \eta^{(1)}, \ldots, \eta^{\left(N_{d}\right)}\right\},
$$

where $x^{(d)}=D^{-1}\left(1-\sum_{k} u^{(k)} u^{(k) \dagger}\right) \eta^{(d)}$.

From the $v$ and $w$ vectors, we may construct meson fields at a temporal coordinate $t$ with the Dirac matrix $\Gamma$ and spatial momentum $\mathbf{p}$

$$
\mathscr{O}_{\Gamma, \phi}^{(k, l)}(t ; \mathbf{p})=\sum_{\mathbf{x}, \mathbf{r}} \phi(\mathbf{r}) w(\mathbf{x}+\mathbf{r}, t)^{(k) \dagger} \Gamma v(\mathbf{x}, t)^{(l)} e^{-i \mathbf{p x}} .
$$

For the smearing function $\phi(\mathbf{r})$, we choose the local $\phi_{l}(\mathbf{r})=\delta_{\mathbf{r}, \mathbf{0}}$ and exponential function $\phi_{S}(\mathbf{r})=$ $\exp [-0.4|\mathbf{r}|]$. Connected and disconnected three-point functions as well as the subtraction term of the vev contribution to the scalar form factor, shown in Fig. 1, are calculated from these meson fields as

$$
\begin{gathered}
C_{\pi \Gamma \pi}^{(\mathrm{conn})}\left(\Delta t, \Delta t^{\prime} ; \mathbf{p}, \mathbf{p}^{\prime}\right)=\frac{1}{N_{t}} \sum_{t} \sum_{k, l, m=1}^{N_{\mathrm{vec}}} \mathscr{O}_{\gamma_{5}, \phi_{s}}^{(m, l)}\left(t+\Delta t+\Delta t^{\prime} ; \mathbf{p}^{\prime}\right) \\
\times \mathscr{O}_{\Gamma, \phi_{l}}^{(l, k)}\left(t+\Delta t ; \mathbf{p}-\mathbf{p}^{\prime}\right) \\
\times \mathscr{O}_{\gamma_{5}, \phi_{s}}^{(k, m)}(t ;-\mathbf{p}),
\end{gathered}
$$



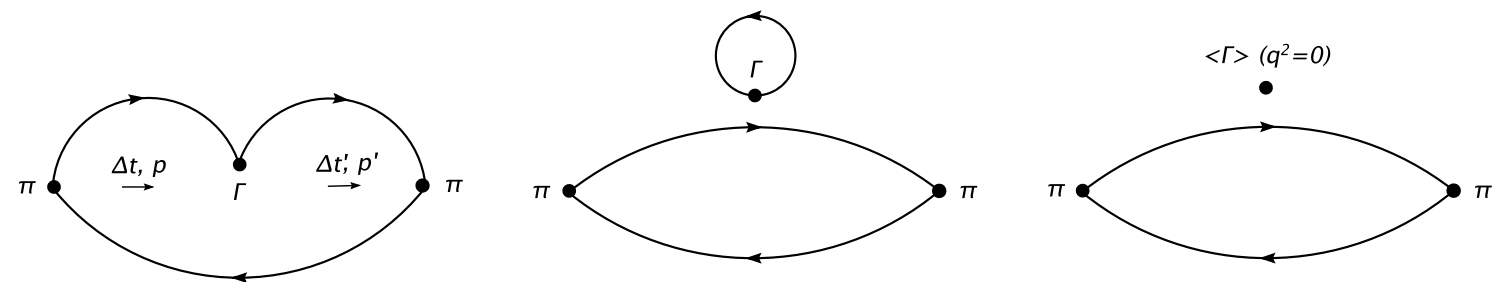

Figure 1: Connected (left-most diagram) and disconnected (middle diagram) three point functions. Note that we have the contribution to $F_{S}(0)$ from the right-most diagram due to the non-zero vacuum expectation value of the scalar operator $S$.

$$
\begin{aligned}
C_{\pi \Gamma \pi}^{(\mathrm{disc})}\left(\Delta t, \Delta t^{\prime} ; \mathbf{p}, \mathbf{p}^{\prime}\right)=\frac{1}{N_{t}} \sum_{t} \sum_{k, l=1}^{N_{\text {vec }}} \mathscr{O}_{\gamma_{5}, \phi_{s}}^{(k, l)}(t+\Delta t & \left.+\Delta t^{\prime} ; \mathbf{p}^{\prime}\right) \mathscr{O}_{\gamma_{5}, \phi_{s}}^{(l, k)}(t ;-\mathbf{p}) \\
& \times \sum_{m=1}^{N_{\text {vec }}} \mathscr{O}_{\Gamma, \phi_{l}}^{(m, m)}\left(t+\Delta t ; \mathbf{p}-\mathbf{p}^{\prime}\right), \\
C_{\pi \Gamma \pi}^{(\mathrm{vev})}\left(\Delta t, \Delta t^{\prime} ; \mathbf{p}, \mathbf{p}^{\prime}\right)=\frac{1}{N_{t}} \sum_{t} \sum_{k, l=1}^{N_{\text {vec }}} \mathscr{O}_{\gamma_{5}, \phi_{s}}^{(k, l)}(t+\Delta t & \left.+\Delta t^{\prime} ; \mathbf{p}^{\prime}\right) \mathscr{O}_{\gamma_{5}, \phi_{s}}^{(l, k)}(t ;-\mathbf{p}) \\
& \times\left\langle\frac{1}{N_{t}} \sum_{t^{\prime}}^{N_{\text {vec }}} \sum_{m=1}^{\text {vec }} \mathscr{O}_{\Gamma, \phi_{l}}^{(m, m)}\left(t^{\prime} ; \mathbf{p}-\mathbf{p}^{\prime}\right)\right\rangle_{\mathrm{conf}},
\end{aligned}
$$

where $\langle\cdots\rangle_{\text {conf }}$ represents a Monte Carlo average. We denote the temporal separation and spatial momentum for the initial (final) meson by $\Delta t$ and $\mathbf{p}\left(\Delta t^{\prime}\right.$ and $\left.\mathbf{p}^{\prime}\right)$, respectively.

Our measurements are carried out at four values of the quark mass $m_{u d}$ in the range $290 \lesssim$ $M_{\pi}[\mathrm{MeV}] \lesssim 520$. We explore the region of the momentum transfer $-1.7 \lesssim q^{2}\left[\mathrm{GeV}^{2}\right] \leq 0$ by taking the meson momentum $\mathbf{p}$ with $|\mathbf{p}| \leq 2$. Note that the spatial meson momentum is shown in units of $2 \pi a / L$ in this article. While we have simulated only the trivial topological sector, the effect of the fixed global topology is suppressed by the inverse of the space-time volume $\sim 1 / V$ [3].

\section{Determination of pion form factors}

We calculate effective value of the vector form factor from a ratio

$$
\begin{aligned}
F_{V}\left(\Delta t, \Delta t^{\prime} ; q^{2}\right) & =\frac{2 M_{\pi}}{E_{\pi}(|\mathbf{p}|)+E_{\pi}\left(\left|\mathbf{p}^{\prime}\right|\right)} \frac{R_{V}\left(\Delta t, \Delta t^{\prime} ;|\mathbf{p}|,\left|\mathbf{p}^{\prime}\right|, q^{2}\right)}{R_{V}\left(\Delta t, \Delta t^{\prime} ; 0,0,0\right)}, \\
R_{V}\left(\Delta t, \Delta t^{\prime} ;|\mathbf{p}|,\left|\mathbf{p}^{\prime}\right|, q^{2}\right) & =\frac{C_{\pi \gamma_{4} \pi}^{(\mathrm{conn})}\left(\Delta t, \Delta t^{\prime} ; \mathbf{p}, \mathbf{p}^{\prime}\right)}{C_{\pi \pi, \phi_{s} \phi_{l}}(\Delta t ; \mathbf{p}) C_{\pi \pi, \phi_{l} \phi_{s}}\left(\Delta t^{\prime} ; \mathbf{p}^{\prime}\right)} .
\end{aligned}
$$

Here $C_{\pi \pi, \phi \phi^{\prime}}$ is the pion two-point function with the smearing function $\phi\left(\phi^{\prime}\right)$ for the source (sink) operator, and it can also be calculated from the meson field Eq. (2.2). We take the average of $R_{V}$ over momentum configurations corresponding to the same value of $q^{2}$. This average as well as that over the location of the source operator in Eqs. (2.3) - (2.5) leads to an accurate estimate of $F_{V}\left(\Delta t, \Delta t^{\prime} ; q^{2}\right)$ as shown in Fig. 2. The vector form factor $F_{\pi}\left(q^{2}\right)$ is determined from a constant fit in a range of $\left(\Delta t, \Delta t^{\prime}\right)$, where $F_{V}\left(\Delta t, \Delta t^{\prime} ; q^{2}\right)$ shows a reasonable plateau. We include the leading finite volume correction (FVC) [7] to $F_{V}\left(q^{2}\right)$.

The scalar form factor normalized at a certain momentum transfer $q_{\mathrm{ref}}^{2}$ can be calculated from a similar ratio 

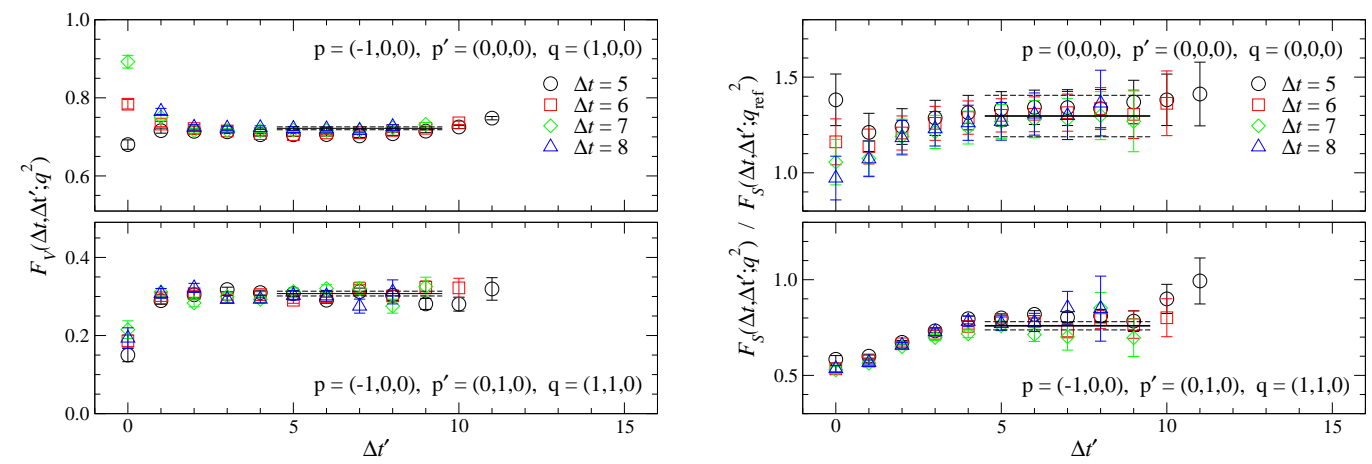

Figure 2: Effective value of $F_{V}\left(\Delta t, \Delta t^{\prime} ; q^{2}\right)$ (left panels) and $F_{S}\left(\Delta t, \Delta t^{\prime} ; q^{2}\right) / F_{S}\left(\Delta t, \Delta t^{\prime} ; q_{\text {ref }}^{2}\right)$ (right panels) at $m_{u d} \sim m_{s, \text { phys }} / 2$, where $m_{s, \text { phys }}$ is the physical strange quark mass.

$$
\frac{F_{S}\left(\Delta t, \Delta t^{\prime} ; q^{2}\right)}{F_{S}\left(\Delta t, \Delta t^{\prime} ; q_{\mathrm{ref}}^{2}\right)}=\frac{R_{S}\left(\Delta t, \Delta t^{\prime} ; q^{2}\right)}{R_{S}\left(\Delta t, \Delta t^{\prime} ; q_{\mathrm{ref}}^{2}\right)}, \quad R_{S}\left(\Delta t, \Delta t^{\prime} ; q^{2}\right)=\frac{C_{\pi 1 \pi}\left(\Delta t, \Delta t^{\prime} ; \mathbf{p}, \mathbf{p}^{\prime}\right)}{C_{\pi \pi, \phi_{s} \phi_{l}}(\Delta t ; \mathbf{p}) C_{\pi \pi, \phi_{l} \phi_{s}}\left(\Delta t^{\prime} ; \mathbf{p}^{\prime}\right)},
$$

where $C_{\pi 1 \pi}=C_{\pi 1 \pi}^{(\mathrm{conn})}-C_{\pi 1 \pi}^{(\mathrm{disc})}+C_{\pi 1 \pi}^{(\mathrm{vev})}$. As Fig. 2 indicates, $F_{S}\left(q^{2}\right)$ at $q^{2}=0$ suffers from a relatively large statistical error than those at $q^{2} \neq 0$ due to the severe cancellation between $C_{\pi 1 \pi}^{\text {(disc) }}$ and $C_{\pi 1 \pi}^{(\mathrm{vev})}$. We therefore use $F_{S}\left(q^{2}\right)$ normalized at the smallest non-zero momentum transfer with $\left|\mathbf{q}_{\text {ref }}\right|=1$ in the following analysis. The normalized form factor $F_{S}\left(q^{2}\right) / F_{S}\left(q_{\mathrm{ref}}^{2}\right)$ is determined by a constant fit, while $\mathrm{FVC}$ to $F_{S}\left(q^{2}\right)$ is not available so far and is not taken into account.

\section{Parametrization of $q^{2}$ dependence}

The vector and scalar form factors are plotted as a function of $q^{2}$ in Fig. 3. We observe that $F_{V}\left(q^{2}\right)$ is close to the pole dependence $1 /\left(1-q^{2} / M_{\rho}^{2}\right)$ with $M_{\rho}$ measured at simulated $m_{u d}$. Its $q^{2}$ dependence is therefore parametrized by the following form of the $\rho$ pole with a polynomial correction to determine the charge radius $\left\langle r^{2}\right\rangle_{V}$ and the curvature $c_{V}$

$$
F_{V}\left(q^{2}\right)=\frac{1}{1-q^{2} / M_{\rho}^{2}}+c_{1} q^{2}+c_{2}\left(q^{2}\right)^{2}+c_{3}\left(q^{2}\right)^{3}=1+\frac{1}{6}\left\langle r^{2}\right\rangle_{V} q^{2}+c_{V}\left(q^{2}\right)^{2}+\cdots
$$

Because the deviation from the $\rho$ pole is small, we obtain a reasonable $\chi^{2} /$ dof $\sim 1$, and results for $\left\langle r^{2}\right\rangle_{V}$ and $c_{V}$ are stable against the inclusion of the cubic correction term.
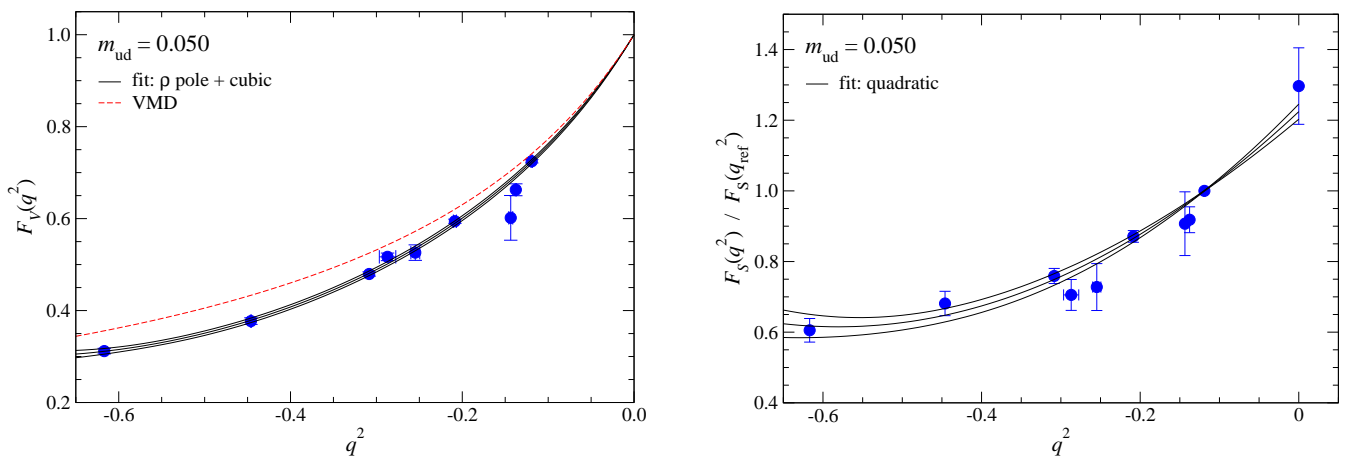

Figure 3: Vector (left panel) and normalized scalar form factors (right panel) at $m_{u d} \sim m_{s, \text { phys }} / 2$ as a function of $q^{2}$. Solid lines show our parametrization and its statistical error. In the left panel, we also plot $\rho$ pole contribution expected from the vector meson dominance hypothesis by the dashed line. 

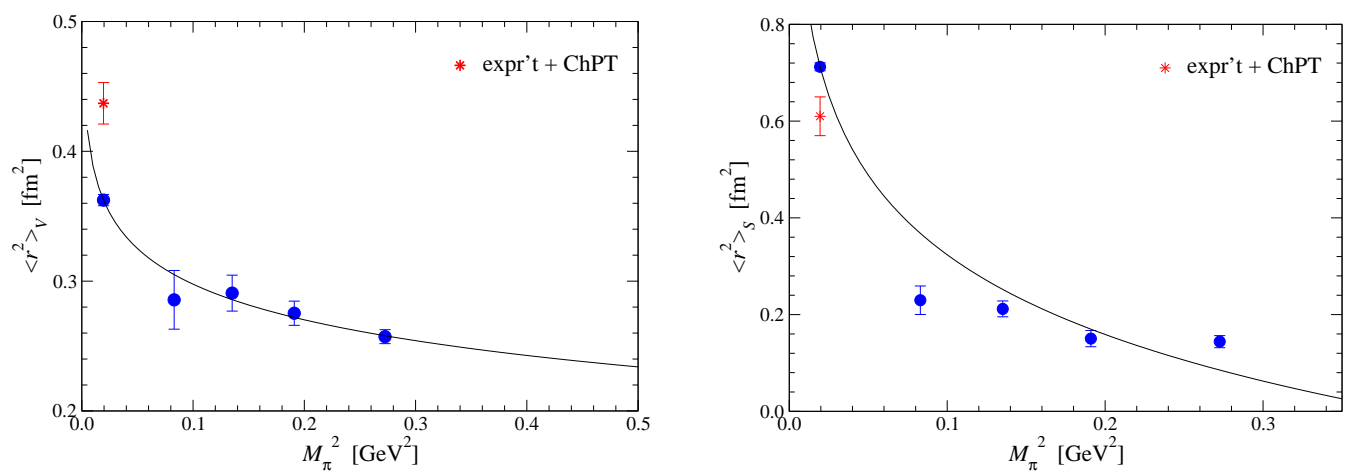

Figure 4: Chiral extrapolation of $\left\langle r^{2}\right\rangle_{V}$ (left panel) and $\left\langle r^{2}\right\rangle_{S}$ (right panel) based on one-loop ChPT. Star symbols show the experimental value for $\left\langle r^{2}\right\rangle_{V}[1]$ and an indirect determination of $\left\langle r^{2}\right\rangle_{S}$ through $\pi \pi$ scattering [10].

Such pole contribution in the scalar channel is not clear within our statistical accuracy. Our data can be fitted to a simple quadratic form

$$
F_{S}\left(q^{2}\right)=F_{S}(0)\left(1+\frac{1}{6}\left\langle r^{2}\right\rangle_{S} q^{2}+c_{S}\left(q^{2}\right)^{2}\right) .
$$

We confirm that the result for the scalar radius $\left\langle r^{2}\right\rangle_{S}$ is stable if we switch to the cubic or single pole form $F_{S}(0) /\left(1-q^{2} / M_{\text {fit }}^{2}\right)$ with $M_{\text {fit }}$ as a fit parameter. The curvature $c_{S}$ is however strongly depends on the choice of the parametrization form, and hence is not used in the following analysis.

\section{Chiral extrapolation}

In one-loop ChPT, the radii $\left\langle r^{2}\right\rangle_{V}$ and $\left\langle r^{2}\right\rangle_{S}$ are given by [8]

$$
\begin{aligned}
\left\langle r^{2}\right\rangle_{V} & =-\frac{1}{N F^{2}}\left(1+6 N l_{6}^{r}\right)-\frac{1}{N F^{2}} \ln \left[\frac{M_{\pi}^{2}}{\mu^{2}}\right], \\
\left\langle r^{2}\right\rangle_{S} & =\frac{1}{N F^{2}}\left(-\frac{13}{2}+6 N l_{4}^{r}\right)-\frac{6}{N F^{2}} \ln \left[\frac{M_{\pi}^{2}}{\mu^{2}}\right],
\end{aligned}
$$

where $N=(4 \pi)^{2}$. We set the renormalization scale $\mu$ to $4 \pi F$, and fix $F$ to the value determined from our study of the pion mass and decay constant [9]. These fits are however not quite successful as seen in Fig. 4. While our data of $\left\langle r^{2}\right\rangle_{V}$ are fitted with reasonable $\chi^{2} /$ dof $\sim 0.3$, the value extrapolated to the physical quark mass $0.362(4) \mathrm{fm}^{2}$ is significantly smaller than experiment $0.437(16) \mathrm{fm}^{2}$ [1]. On the other hand, the one-loop formula for $\left\langle r^{2}\right\rangle_{S}$ with the enhanced chiral $\log$ fails to reproduce our data and results in large $\chi^{2} /$ dof $\sim 16$. We note that similar mild quark mass dependence of the radii is also observed by the ETM Collaboration with a different discretization on a slightly finer lattice [11]. It is unlikely that the failure of the fits within one-loop ChPT is caused by systematic uncertainties due to the fixed topology and the finite lattice spacing.

We then extend our analysis to two loops. The higher order contributions to the radii are given by [1]

$$
\Delta\left\langle r^{2}\right\rangle_{V}=\frac{1}{N^{2} F^{4}}\left(\frac{13 N}{192}-\frac{181}{48}+6 N^{2} r_{V, 1}^{r}\right) M_{\pi}^{2}+\frac{1}{N^{2} F^{4}}\left(\frac{19}{6}-12 N l_{1,2}^{r}\right) M_{\pi}^{2} \ln \left[\frac{M_{\pi}^{2}}{\mu^{2}}\right]
$$



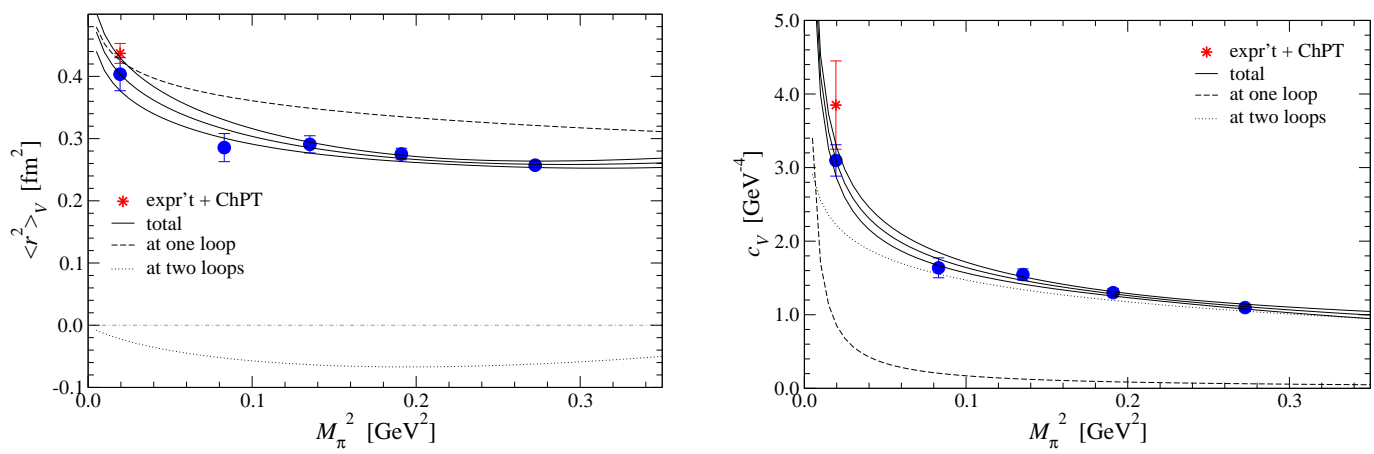

Figure 5: Simultaneous chiral extrapolation of $\left\langle r^{2}\right\rangle_{V}$ (left panel) and $c_{V}$ (right panel) based on two-loop ChPT (solid lines). We also plot one- and two-loop contributions by dashed and dotted lines, respectively.

$$
\begin{aligned}
\Delta\left\langle r^{2}\right\rangle_{S}= & \frac{1}{N^{2} F^{4}}\left(-\frac{23 N}{192}+\frac{869}{108}+88 N l_{1,2}^{r}+80 N l_{2}^{r}+5 N l_{3}^{r}-24 N^{2} l_{3}^{r} l_{4}^{r}+6 N^{2} r_{S, 1}^{r}\right) M_{\pi}^{2} \\
& +\frac{1}{N^{2} F^{4}}\left(-\frac{323}{36}+124 N l_{1,2}^{r}+130 N l_{2}^{r}\right) M_{\pi}^{2} \ln \left[\frac{M_{\pi}^{2}}{\mu^{2}}\right]-\frac{65}{3 N^{2} F^{4}} M_{\pi}^{2} \ln \left[\frac{M_{\pi}^{2}}{\mu^{2}}\right]^{2} .
\end{aligned}
$$

At two loops, the curvature $c_{V}$ has non-trivial contributions and can be included in our analysis

$$
\begin{aligned}
c_{V}= & \frac{1}{60 N F^{2}} \frac{1}{M_{\pi}^{2}}+\frac{1}{N^{2} F^{4}}\left(\frac{N}{720}-\frac{8429}{25920}+\frac{N}{3} l_{1,2}^{r}+\frac{N}{6} l_{6}^{r}+N^{2} r_{V, 2}^{r}\right) \\
& +\frac{1}{N^{2} F^{4}}\left(\frac{1}{108}+\frac{N}{3} l_{1,2}^{r}+\frac{N}{6} l_{6}^{r}\right) \ln \left[\frac{M_{\pi}^{2}}{\mu^{2}}\right]+\frac{1}{72 N^{2} F^{4}} \ln \left[\frac{M_{\pi}^{2}}{\mu^{2}}\right]^{2} .
\end{aligned}
$$

The analytic terms containing $r_{X, i}^{r}(X=V, S, i=1,2)$ represent contributions from $O\left(p^{6}\right)$ chiral Lagrangian. We denote the linear combination $l_{1}^{r}-l_{2}^{r} / 2$ appearing commonly in $\left\langle r^{2}\right\rangle_{V}$ and $c_{V}$ as $l_{1,2}^{r}$.

While the two-loop formulae involve many LECs, the simultaneous fit to $\left\langle r^{2}\right\rangle_{V}$ and $c_{V}$ has only four free parameters $l_{6}^{r}, l_{1,2}^{r}, r_{V, 1}^{r}$ and $r_{V, 2}^{r}$. This fit plotted in Fig. 5 shows that the two-loop contributions are significant in our simulated region of $m_{u d}$. We obtain a reasonable value of $\chi^{2} /$ dof $\sim 0.5$, and the extrapolated values of $\left\langle r^{2}\right\rangle_{V}$ and $c_{V}$ are consistent with experiment [1].

The inclusion of $\left\langle r^{2}\right\rangle_{S}$ into the simultaneous chiral fit introduces additional four free parameters. In order to stabilize this fit, we fix $l_{2}^{r}$ and $l_{3}^{r}$, which appear only in the two-loop terms, to a phe-

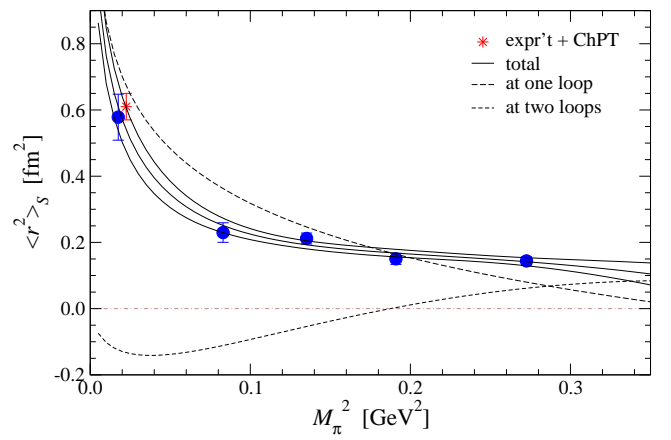

Figure 6: Chiral extrapolation of $\left\langle r^{2}\right\rangle_{S}$ from simultaneous to $\left\langle r^{2}\right\rangle_{V, S}$ and $c_{V}$ based on two-loop formulae. nomenological estimate $\bar{l}_{2}=4.31[10]$ and a lattice result $\bar{l}_{3}=3.44$ from our analysis of the pion spectroscopy [9] ${ }^{1}$. The extrapolation of $\left\langle r^{2}\right\rangle_{V}$ and $c_{V}$ turns out to be consistent with those in Fig. 5. The extrapolation of $\left\langle r^{2}\right\rangle_{S}$ is shown in Fig.6. From this simultaneous fit, we obtain

$$
\left\langle r^{2}\right\rangle_{V}=0.404(22)(22) \mathrm{fm}^{2},\left\langle r^{2}\right\rangle_{S}=0.578(69)(46) \mathrm{fm}^{2}, c_{V}=3.11(14)(86) \mathrm{GeV}^{-4} .
$$

\footnotetext{
${ }^{1}$ The $\mu$ independent convention $\bar{l}_{i}$ is defined from $l_{i}^{r}=\gamma_{i}\left(\bar{l}_{i}+\ln \left[M_{\pi}^{2} / \mu^{2}\right]\right) / 2 N$ with $\gamma_{3}=-1 / 2, \gamma_{4}=2$ and $\gamma_{6}=-1 / 3$.
} 
The first error is statistical. The second is systematic error estimated by changing the inputs for $l_{2}^{r}$ and $l_{3}^{r}$ to different phenomenological estimates in Ref.[1], and by limiting the fitting data to the radii $\left(\left\langle r^{2}\right\rangle_{V}\right.$ and $\left.\left\langle r^{2}\right\rangle_{S}\right)$ or those in the vector channel $\left(\left\langle r^{2}\right\rangle_{V}\right.$ and $\left.c_{V}\right)$. We also test $\left\langle r^{2}\right\rangle_{S}$ from the cubic parametrization for the $q^{2}$ dependence of $F_{S}\left(q^{2}\right)$. Note that all the extrapolated values in Eq. (5.6) are consistent with experiment.

We obtain $\bar{l}_{6}=11.8(0.7)(1.3), \bar{l}_{4}=4.06(44)(99)$, and $l_{1,2}^{r}=-2.9(0.8)(2.4) \times 10^{-3}$ for the $O\left(p^{4}\right)$ LECs. Our estimate of $\bar{l}_{6}$ is slightly smaller than 16.0(0.9) obtained in Ref.[1] partly due to a deviation of $F$ between our lattice determination [9] and two-loop ChPT [12]. We note that $\bar{l}_{4}$ is consistent with our determination $\bar{l}_{4}=4.12(56)$ from $F_{\pi}$ [9] and a phenomenological estimate 4.39(22) [10]. Our results for the $O\left(p^{6}\right)$ LECs are $r_{V, 1}^{r}=-1.1 \times 10^{-5}, r_{V, 2}^{r}=-4.0 \times 10^{-5}$ and $r_{S, 1}^{r}=1.3 \times 10^{-4}$ with substantial uncertainty of $50-100 \%$.

\section{Conclusions}

In this article, we report on our calculation of the pion form factors with two flavors of dynamical overlap quarks. By employing the all-to-all quark propagators, $F_{S}\left(q^{2}\right)$ is calculated including contributions from the disconnected diagrams for the first time. The one-loop ChPT formulae fail to reproduce our data of $F_{S}\left(q^{2}\right)$. In our analysis extended to two loops, we observe significant two-loop contributions at our simulated quark masses, and obtain $\left\langle r^{2}\right\rangle_{V, S}$ and $c_{V}$ consistent with experiment. Further investigations of systematics due to the fixed global topology and quenching of strange quarks are in progress by direct simulations in the non-trivial topological sectors and in three-flavor QCD.

Numerical simulations are performed on Hitachi SR11000 and IBM System Blue Gene Solution at High Energy Accelerator Research Organization (KEK) under a support of its Large Scale Simulation Program (No. 08-05). This work is supported in part by the Grant-in-Aid of the Ministry of Education (No. 18340075, 18740167, 19540286, 19740160, 20025010, 20039005, 20340047, and 20740156), the National Science Council of Taiwan (No. NSC96-2112-M-002-020-MY3, NSC96-2112-M-001-017-MY3, NSC97-2119-M-002-001), and NTU-CQSE (No. 97R0066-69).

\section{References}

[1] J. Bijnens, G. Colangelo and P. Talavera, JHEP 9805, 014 (1998).

[2] T. Kaneko et al. (JLQCD Collaboration), PoS LATTICE 2007, 148.

[3] S. Aoki, H. Fukaya, S. Hashimoto and T. Onogi, Phys. Rev. D 76, 054508 (2007).

[4] S. Aoki et al. (JLQCD Collaboration), Phys. Rev. D 78, 014508 (2008).

[5] H. Matsufuru, PoS LATTICE 2007, 018; S. Hashimoto, in these proceedings.

[6] J. Foley et al. (TrinLat Collaboration), Comput. Phys. Commun, 172, 145 (2005).

[7] B. Borasoy and R. Lewis, Phys. Rev. D 71, 014033 (2005).

[8] J. Gasser and H. Leutwyler, Ann. Phys. 158, 142 (1984).

[9] J. Noaki et al. (JLQCD and TWQCD Collaborations), arXiv:0806.0894 [hep-lat].

[10] G. Colangelo, J. Gasser and H. Leutwyler, Nucl. Phys. B 603, 125 (2001).

[11] S. Simula (ETM Collaboration), PoS LATTICE 2007, 371.

[12] G. Colangelo and S. Dürr, Eur. Phys. J. C 33, 543 (2004). 